\title{
COMMUNITY BASED SCREENING FOR DIABETES AND PREDIABETES USING THE INDIAN DIABETES RISK SCORE AMONG ADULTS IN A SEMI-URBAN AREA IN KATHMANDU, NEPAL
}

\author{
Silvanus V', Dhakal N¹, Pokhrel A², Baral BK', Panta PP1
}

${ }^{1}$ Department of Community Medicine, ${ }^{2}$ Department of Biochemistry, Nepal Medical College Teaching Hospital, Attarkhel, Gokarneshwor-8, Kathmandu, Nepal

\begin{abstract}
Diabetes has been recognized as a "global health emergency" with an estimated $9 \%$ of adults being affected. However, about half of these adults remain undiagnosed. Conventional screening tools like fasting plasma glucose (FPG), oral glucose tolerance testing (OGTT) and glycosylated haemoglobin (HbA1c) can be inconvenient and expensive in a community-based setting. The Indian Diabetes Risk Score (IDRS) is a simple, non-invasive tool which has been validated for use in the Indian population. Age, abdominal obesity, family history of diabetes and physical activity levels have been weighted for a maximum score of 100. Persons with IDRS of $<30$ are categorized as low risk, 30-50 as medium risk and those with $>60$ as high risk for diabetes. A community based, cross-sectional, analytical study was planned to assess the performance of IDRS among adults in a semi-urban area in Kathmandu, Nepal. A total of 256 (170 female, 86 male) persons without diabetes from 260 households were screened during the study period. A majority (46.09\%) were classified as high risk, $44.53 \%$ as moderate risk and $9.38 \%$ as low risk for developing diabetes. Among them, $162(63.28 \%)$ volunteered for definitive testing. The prevalence of undiagnosed diabetes and prediabetes was $4.32 \%$ (95\% CI: $1.75 \%$ to $8.70 \%$ ) and $7.14 \%$ (95\% CI: $3.89 \%$ to $12.58 \%$ ) respectively. IDRS predicted the combined risk of diabetes and prediabetes with sensitivity of $84.21 \%$ and specificity of $55.24 \%$ in adults with score of 60 and above. The area under the ROC curve (AUC) of IDRS for identifying diabetes and prediabetes was 0.69 as compared to the gold standard (2hour Plasma Glucose) AUC of 0.98. IDRS may be a suitable screening tool for diabetes and prediabetes in the adult Nepalese study population.
\end{abstract}

\section{KEYWORDS}

Diabetes, IDRS, prediabetes, screening, sensitivity, specificity

\section{CORRESPONDING AUTHOR}

Dr. Vinutha Silvanus,

Assistant Professor, Department of Community Medicine, Nepal Medical College Teaching Hospital,

Attarkhel, Gokarneshwor-8, Kathmandu, Nepal,

Email: silvanus.v@gmail.com 


\section{INTRODUCTION}

Diabetes has been recognized as "one of the largest global health emergencies of the 21st century". ${ }^{1}$ Type 2 diabetes (T2D) accounts for up to $90 \%$ of diabetes worldwide. $^{2}$

Globally, $9 \%$ of adults are estimated to have diabetes, with 1 in 2 adults being undiagnosed. Up to 80\% of individuals with undiagnosed diabetes are believed to live in low and middle income countries. ${ }^{3}$

Diagnosed T2D is only the tip of the iceberg of an epidemic of glucose intolerance. Defects in the action or secretion of insulin are the two major abnormalities leading to development of glucose intolerance. The natural history of T2D includes a preceding period of impaired glucose tolerance (IGT) or impaired fasting glucose (IFG). ${ }^{4}$ Prediabetes (IFG and IGT) refers to this stage between normal blood glucose levels and diabetes. They represent persons who are at risk of developing diabetes mellitus in the future. ${ }^{5}$

Diabetes screening is a search for undiagnosed diabetes as well as to identify those who are at risk of developing diabetes. The choice of a screening method should be made on the basis of prevalence of undiagnosed diabetes in the community, the accuracy and cost of the test. ${ }^{6}$

The fasting plasma glucose (FPG), oral glucose tolerance test (OGTT) and glycated haemoglobin are commonly used tests in high resource settings. ${ }^{7}$ In the community setting, random capillary blood glucose (RCBG) and urine glucose testing may provide a good compromise between accuracy and cost effectiveness. ${ }^{8}$ Diabetes risk scores are simple, fast, inexpensive, non- invasive and reliable screening tools. They utilize specific variables or risk factors to calculate an individuals' risk of developing diabetes. They do not quantify the risk of diabetes but help to identify individuals at high risk in a time gap between 5-10 years. ${ }^{9}$

The Indian Diabetes Risk Score (IDRS) is comprised of four scored questions regarding age, abdominal obesity as measured by the waist circumference, family history of diabetes and physical activity levels. It is a simple, easy to administer screening tool to detect undiagnosed diabetes and to identify persons at risk of developing diabetes. ${ }^{10}$

The IDRS validation was carried out among the Chennai Urban and Rural Epidemiological study (CURES) population. An IDRS value of $\geq 60$ was recommended as the cut-off to classify persons as high risk. Persons with high risk classification can be recommended to undergo definitive testing. IDRS was proposed to make screening programmes in India more cost effective. ${ }^{10}$

A systematic review of risk scores or prediction models has suggested that such tools often work well in the population that they have been validated in. The predictive values of risk scores can vary widely between populations. Hence, prior to utilization of a tool, the ease of implementation, cut off points and validation of the risk score in a proposed population needs to be considered. ${ }^{9}$

A systematic review and meta-analysis on the prevalence of T2D in Nepal reported a minimum of $1.4 \%$ to a maximum of $19.0 \%$ with a pooled prevalence of $8.4 \%$. The prevalence of type 2 diabetes in urban and rural populations was $8.1 \%$ (95\% CI: $7.3-8.9 \%)$ and 1.0\% (95\% CI: $0.7-1.3 \%)$, respectively. ${ }^{11}$

This study was planned to assess the prevalence of undiagnosed type 2 diabetes, identify those who are at high risk of developing T2D (prediabetes) in the community and assess the performance of the IDRS as a screening tool among an adult population in a semi-urban area in Kathmandu, Nepal.

\section{MATERIALS AND METHODS}

A community-based cross-sectional, observational and analytical study was carried out in the Budhanilkantha Municipality from September 2017 to May 2018.

Assuming a baseline prevalence of diabetes in Nepal of $8.4 \%$ as an equivalent proportion of undiagnosed diabetes, a $5 \%$ margin of error and a non-response rate of $20 \%$, the minimum sample size was calculated to be 142 adults who were not previously diagnosed with diabetes.

For assessing the performance of IDRS, all of the study participants who were not previously diagnosed with diabetes were invited for an oral glucose tolerance test (OGTT) (WHO 1998 criteria) for definitive testing.

Sampling technique: Using a simple random sampling technique, 260 households were selected for a family survey from an estimated 600 households in the study area. Data collection was carried out in a step wise manner which increased the initial response rate for screening for diabetes and helped to acquire the number needed to undertake definite testing. ${ }^{12}$

Step 1: Adults over 20 years of age who were present in the household and not pregnant at the time of the survey were interviewed using a pretested structured questionnaire (modified STEPS survey) and IDRS screening tool after obtaining due written consent by trained UG medical students supervised by faculty from the department of Community Medicine. $^{13}$

Step 2: Physical measurement of height, weight, waist circumference and hip circumference was carried out at the household level as a part of the family study assessment.

Step 3: Estimation of the random capillary plasma glucose (RCBG) levels was offered to all the 
participants using a glucometer at the household level. Faculty and trained UG students carried out this testing during the study period.

Step 4: All of the study participants were invited to undergo an OGTT as recommended by the WHO guidelines using $75 \mathrm{~g}$ of glucose. ${ }^{5}$ Fasting plasma glucose (FPG) and 2-hour plasma glucose (2 hr PG) samples were collected through a screening camp organized over 7 days in the community. Faculty from the departments of Community Medicine and Biochemistry conducted the screening camp along with the co-operation of community leaders and representatives, screened households and UG medical students. The volunteers were advised to consume the last meal by $8 \mathrm{pm}$ so that they were fasting for a minimum of 10 hours at the time of the testing.

The camp began at 6:45 am in the morning at a predetermined venue in the community considering the convenience of the volunteers. Fasting blood samples were collected by 8 a.m. and all the 2-hour OGTT samples were collected by 10 a.m. The volunteers were advised to rest and refrain from strenuous activities during the testing period and to report any adverse effects following glucose administration.

\section{Oral Glucose Tolerance Test}

A fasting sample of venous blood was collected in a fluoride containing tube. The volunteers were given 75 grams of glucose dissolved in water (about 250-300 ml). The time of oral glucose administration was noted. Another venous blood sample was collected in a fluoride containing tube after 2 hours of glucose loading.
Collected venous blood samples were stored in a lab specimen transport bag and kept protected from direct sunlight. All specimens collected were delivered to the laboratory within 4 hours of collection for the separation of plasma from the cells. The samples were centrifuged at $3000 \mathrm{rpm}$ for 15 minutes and were estimated for plasma glucose by autoanalyzer (Johnson \& Johnson Vitros 250, USA) using glucose oxidase method in the laboratory of NMCTH.

Using the WHO 1999 consulting group criteria, diabetes was defined as FPG $\geq 126 \mathrm{mg} / \mathrm{dl}$ or $2 \mathrm{hr}$ PG $\geq 200 \mathrm{mg} / \mathrm{dl}$. Prediabetes was defined as two categories, impaired fasting glucose (IFG) as FPG $\geq 110$ and $<126 \mathrm{mg} / \mathrm{dl}$ with $2 \mathrm{hr}$ PG $<140 \mathrm{mg} /$ dl. Impaired glucose tolerance (IGT), the second category in prediabetes was defined as FPG $<110 \mathrm{mg} /$ $\mathrm{dl}$ and $2 \mathrm{hr}$ PG $\geq 140$ and $<200 \mathrm{mg} / \mathrm{dl}$. Prediabetes and diabetes were collectively labelled as 'Raised blood glucose'. Normoglycemia was defined as FPG <110 $\mathrm{mg} / \mathrm{dl}$ and $2 \mathrm{hr}$ PG $<140 \mathrm{mg} / \mathrm{dl}^{3,5}$

Persons with previously diagnosed diabetes during the screening period were labelled as 'known diabetes'.

A total of 306 persons were screened during the study period. Fifty persons were found to be previously diagnosed cases of diabetes. The study participants without diabetes $(n=256)$ were invited to undergo an OGTT. One hundred and sixty-two participants $(63.28 \%)$ responded to our invitation. One hundred and fifty-nine participants underwent the OGTT. Three participants were tested for plasma glucose levels after a meal (post prandial) as they either vomited or were unable to drink the glucose solution. (Flowchart 1)

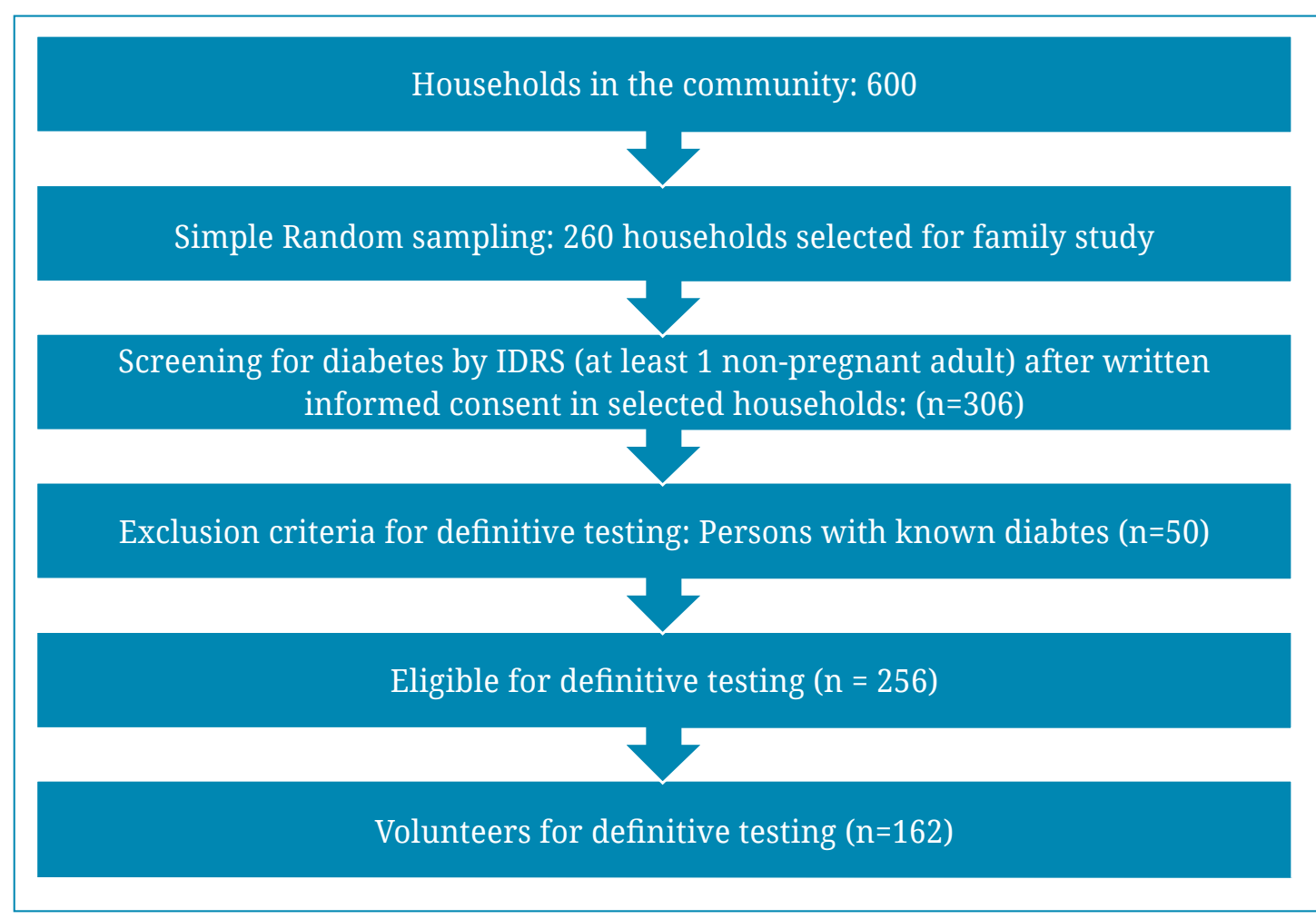

Flow Chart 1: Sampling and screening of Volunteers 
DATA COLLECTION TOOL: The IDRS had four scored risk factors: age, abdominal obesity, family history of diabetes and physical activity levels. A maximum score of 100 was given for these combined categories.

Subjects with an IDRS of $<30$ were categorized as low risk, 30-50 as medium risk and those with $\geq 60$ as high risk for diabetes.

Validity of the tool: An IDRS value > or $=60$ had the optimum sensitivity (72.5\%) and specificity (60.1\%) for determining undiagnosed diabetes with a positive predictive value of $17.0 \%$, negative predictive value of $95.1 \%$, and accuracy of $61.3 \%{ }^{10}$

Data Analysis: Collected data was entered in EPIINFO and data was analysed using Stata 15IC licensed software.

The prevalence rate of known and undiagnosed (screen-detected) diabetes and prediabetes (raised blood glucose) was reported with 95\% confidence intervals. Association between categorical data and IDRS classification was analysed using the Chisquare test or Fisher exact test. RCBG, FPG, 2hr PG were reported as the median with interquartile range. Normally distributed quantitative variables were reported as means with standard deviation.

IDRS risk score and blood glucose concentration levels: Receiver operator characteristic (ROC) curves were constructed to determine the sensitivity and specificity of IDRS score cut-off values for identifying diabetes and prediabetes. The area under the ROC curve for IDRS >= 60 was constructed in comparison to the gold standard 2-hour PG and FPG.

Ethical clearance for the study was obtained from the Nepal Medical College Institutional Review Committee (NMC IRC).

\section{RESULTS}

Fifty persons were found to be previously diagnosed cases of diabetes, the prevalence of known diabetes (50/ 306) was an estimated 16.34\% (95\% CI: 12.62\% to $20.90 \%$ ). (Table-1)

Two hundred and fifty-six (170 female, 86 male) persons without diabetes comprised the IDRS screened population. A majority, 46.09\% were classified as high risk, $44.53 \%$ as moderate risk and $9.38 \%$ as low risk for developing diabetes. (Table-2)

An item-wise analysis of the IDRS scored questions has been shown in Table-3. A majority (41.41\%) of the persons screened were 50 years old and above. Abdominal or central obesity was seen in $84.02 \%$ of females and $64.37 \%$ of males. About two thirds $(65.75 \%)$ of the screened participants reported mild physical activity levels at home or work. Only 6.64\% reported that one parent had diabetes while the other participants did not report a family history of diabetes. (Table-3) There was a highly significant association between a high-risk IDRS score and hypertensive status among the screened population $(n=256)$ (chi-square value 8.742 df 1 prob 0.003) (Table-4)

Among those who underwent definitive testing $(n=162)$, mean age was $49.15( \pm 15.12)$ and BMI 26.10 ( \pm 4.08). Median FPG, $2 \mathrm{hr}$ PG and RCBG was $77 \mathrm{mg} / \mathrm{dl}$ (71- 85mg/dl), $81 \mathrm{mg} / \mathrm{dl}$ (64-107mg/dl) and $102 \mathrm{mg} / \mathrm{dl}$ (90-121 mg/dl) respectively. All of the anthropometric measures, mean age and median values of plasma glucose and random capillary blood glucose were significantly higher among those with a high-risk classification. ( $<$ < 0.05). (Table-5 \&6)

The prevalence of undiagnosed diabetes was $4.32 \%$ (95\% CI $1.75 \%$ to $8.70 \%)$ and that of prediabetes was $7.14 \%$ (95\% CI $3.89 \%$ to $12.58 \%)$. The overall

\begin{tabular}{|lccccc|}
\hline \multicolumn{5}{c}{ Table-1: Prevalence of 'known Diabetes' during community-based screening period } \\
Diabetes & Frequency & Percent & Cum. Percent & Wilson 95\% LCL & $\begin{array}{c}\text { Wilson 95\% } \\
\text { UCL }\end{array}$ \\
Yes & 50 & $16.34 \%$ & $16.34 \%$ & $12.62 \%$ & $20.90 \%$ \\
No & 256 & $83.66 \%$ & $100.00 \%$ & $79.10 \%$ & $87.38 \%$ \\
TOTAL & $\mathbf{3 0 6}$ & $\mathbf{1 0 0 . 0 0 \%}$ & $\mathbf{1 0 0 . 0 0 \%}$ & & \\
\hline
\end{tabular}

(LCL Lower confidence limit, UCL Upper confidence limit)

Table-2: Risk of diabetes (IDRS score) categorized by gender

\begin{tabular}{lccccc|} 
Sex & Low risk $(<\mathbf{3 0})$ & Moderate risk (30-50) & High risk $(\geq \mathbf{6 0 )}$ & TOTAL & Chi-square Prob \\
Female & 15 & 74 & 81 & 170 & 0.5431 \\
Male & 9 & 40 & 37 & 86 & df 2 \\
TOTAL & $\mathbf{2 5 ( 9 . 3 8 \% )}$ & $\mathbf{1 1 2 ( 4 4 . 5 3 \% )}$ & $\mathbf{1 1 9}(\mathbf{4 6 . 0 9 \% )}$ & $\mathbf{2 5 6}$ & $\mathrm{p}=0.76 \mathrm{NS}$ \\
\hline
\end{tabular}


Table-3: Item-wise analysis of the IDRS among the screened population ( $\mathrm{n}=256)$

\begin{tabular}{lcccc} 
IDRS & Score & Frequency & Percent & Exact 95\% CI \\
Age classification (n=256) & & & & \\
$<35$ years & 0 & 47 & $18.4 \%$ & $13.81-23.66$ \\
35 - 49 years & 20 & 103 & $40.2 \%$ & $34.18-46.52$ \\
$\geq 50$ years & 30 & 106 & $41.4 \%$ & $35.31-47.71$ \\
Waist Circumference (Female) Classification (n=170) & & & & \\
$<80$ cm & 0 & 28 & $16.0 \%$ & $10.80-22.39$ \\
80 to 89 cm & 10 & 67 & $40.0 \%$ & $32.22-47.44$ \\
90 cm or more & 20 & 75 & $44.4 \%$ & $36.75-52.21$ \\
Waist Circumference (Male) Classification (n=86) & & & & \\
$<90$ cm & 0 & 32 & $37.2 \%$ & $25.65-46.62$ \\
90 to 99 cm & 10 & 32 & $37.2 \%$ & $26.69-47.80$ \\
100 cm or more & 20 & 22 & $25.6 \%$ & $18.54-38.21$ \\
Physical Activity levels (n=256) & & & & \\
No exercise or sedentary activities & & & & \\
Regular mild exercise or physical activity & 0 & 22 & $9.1 \%$ & $5.83-13.28$ \\
Regular moderate exercise or manual activity & 10 & 166 & $64.9 \%$ & $59.56-71.57$ \\
Regular strenuous exercise or manual activity & 20 & 68 & $26.6 \%$ & $19.98-31.0$ \\
Family History of Diabetes (n=256) & 30 & 0 & 0 & 0 \\
No diabetes in parents & & & & \\
One parent has diabetes & & & & \\
Both parents have diabetes & 0 & 239 & $93.4 \%$ & $89.58-96.08$ \\
TOTAL & 10 & 17 & $6.7 \%$ & $3.92-10.42$ \\
\hline
\end{tabular}

Table-4: IDR Risk Classification categorized by the hypertensive status of the screened population

Hypertension

IDRS Risk Classification

Chi-square test Probability Low to Moderate risk High risk Total

\begin{tabular}{lcccc} 
Yes & 13 & 27 & 40 & 8.742 df 1 \\
No & 125 & 91 & 216 & prob 0.003 \\
Total & $\mathbf{1 3 7}$ & $\mathbf{1 1 9}$ & $\mathbf{2 5 6}$ & \\
\hline
\end{tabular}

\section{Table-5: Mean values of age and BMI among the definitive testing volunteers $(n=162)$}

\begin{tabular}{|lccccc} 
Variable & & $\begin{array}{c}\text { High risk } \\
(\mathbf{n}=79)\end{array}$ & $\begin{array}{c}\text { Low to moderate } \\
\text { risk }(\mathbf{n}=\mathbf{8 3})\end{array}$ & $\begin{array}{c}\text { Mean Difference } \\
\mathbf{( 9 5 \% ~ C I )}\end{array}$ & $\begin{array}{c}\text { Independent } \\
\text { t test p value }\end{array}$ \\
Age (years) & $49.15( \pm 15.12)$ & $57.40( \pm 12.66)$ & $41.31( \pm 12.97)$ & $16.10(12.12-20.08)$ & 0.000 \\
$\begin{array}{l}\mathrm{BMI}\left(\mathrm{kg} / \mathrm{m}^{2}\right) \\
\mathrm{n}=157\end{array}$ & $26.10( \pm 4.08)$ & $27.56( \pm 4.29)$ & $24.72( \pm 3.52)$ & $8.18(7.53-8.8)$ & 0.000 \\
\hline
\end{tabular}

(BMI: Body Mass Index) 


\begin{tabular}{|c|c|c|c|c|c|}
\hline \multirow{2}{*}{ Variable } & \multicolumn{2}{|c|}{ OGTT respondents $(n=162)$} & \multirow{2}{*}{$\begin{array}{l}\text { High risk (n=80) } \\
\text { Median (IQR) }\end{array}$} & \multirow{2}{*}{$\begin{array}{l}\text { Low to moderate } \\
\text { risk }(\mathrm{n}=82) \\
\text { Median (IQR) }\end{array}$} & \multirow{2}{*}{$\begin{array}{l}\text { Kruskal Wallis } \\
\text { test for two } \\
\text { groups }\end{array}$} \\
\hline & Mean & Median (IQR) & & & \\
\hline FPG (mg/dl) & 82.15 & $77(71-85))$ & $80(72-89)$ & $75(70-80)$ & $\begin{array}{l}\text { 10.92, df } 1 \\
p=0.000\end{array}$ \\
\hline $\begin{array}{l}2 \mathrm{hr} \text { PG (mg/ } \\
\text { dl) }\end{array}$ & 94.59 & $81(64-107)$ & $90(75-128)$ & $73(61-91)$ & $\begin{array}{l}\text { 12.89, df } 1, \\
p=0.0003\end{array}$ \\
\hline $\begin{array}{l}\text { RCBG (mg/dl) } \\
(n=71)\end{array}$ & 114.35 & $102(90-121)$ & $\begin{array}{c}116(99-138) \\
(n=33)\end{array}$ & $\begin{array}{c}104(91-122) \\
(n=38)\end{array}$ & $\begin{array}{l}\text { 4.26, df } 1 \\
p=0.03\end{array}$ \\
\hline
\end{tabular}

(FPG Fasting plasma glucose, 2 hr PG 2-hour plasma glucose, RCBG Random capillary blood glucose)

Table-7: Prevalence of undiagnosed diabetes and prediabetes (Raised blood glucose) among the definitive testing volunteers

\begin{tabular}{|c|c|c|c|c|}
\hline $\begin{array}{l}\text { WHO (1999) Diabetes } \\
\text { Classification }\end{array}$ & Frequency & Percent & Exact $95 \%$ LCL & Exact $95 \%$ UCL \\
\hline Normoglycemia & 143 & 88.27 & $82.29 \%$ & $92.79 \%$ \\
\hline Prediabetes & 12 & 7.41 & $3.89 \%$ & $12.58 \%$ \\
\hline Diabetes & 7 & 4.32 & $1.75 \%$ & $8.70 \%$ \\
\hline TOTAL & 162 & 100.00 & & \\
\hline
\end{tabular}

Table-8: Sensitivity and specificity of IDRS at high risk cut-off score $\geq 60$

\begin{tabular}{|lccccc|} 
IDRS classification & \multicolumn{2}{c}{ Raised Blood Glucose } & Total & $\begin{array}{c}\text { Fisher Exact } \\
\text { P value }\end{array}$ & OR Exact CI \\
High risk & Yes & No & & & \\
& $16(84.21 \%)$ & $64(44.76 \%)$ & $80(49.38 \%)$ & \\
Low to moderate risk & $3(15.79 \%)$ & $79(55.24 \%)$ & $82(50.62 \%)$ & 0.001 & $(1.75-36.41)$ \\
Total & $\mathbf{1 9}$ & $\mathbf{1 4 2}$ & $\mathbf{1 6 2}$ & & \\
\hline
\end{tabular}

(OR Odd's ratio, CI Confidence interval)

Table-9: Receiver Operator Characteristic Curve Sensitivity and Specificity values for IDRS cutpoints

\begin{tabular}{lccccc|} 
IDRS cut point & Sensitivity (\%) & Specificity (\%) & $\begin{array}{c}\text { Correctly } \\
\text { Classified (\%) }\end{array}$ & Positive LR & Negative LR \\
$>=\mathbf{1 0}$ & 100 & 0.00 & 11.73 & 1.00 & \\
$>=\mathbf{2 0}$ & 100 & 2.10 & 13.58 & 1.02 & 0.00 \\
$>=\mathbf{3 0}$ & 100 & 10.49 & 20.99 & 1.11 & 0.00 \\
$>=\mathbf{4 0}$ & 100 & 16.78 & 26.54 & 1.20 & 0.00 \\
$>=\mathbf{5 0}$ & 100 & 27.27 & 35.80 & 1.37 & 0.00 \\
$>=\mathbf{6 0}$ & 84.21 & 55.24 & 58.64 & 1.88 & 0.28 \\
$>=\mathbf{7 0}$ & 31.58 & 86.01 & 79.63 & 2.25 & 0.79 \\
$>=\mathbf{8 0}$ & 15.79 & 98.60 & 88.89 & 11.28 & 0.85 \\
\hline
\end{tabular}

(LR Likelihood ratio) 
prevalence of persons with 'raised blood glucose' was $11.73 \%$ (95\% CI $5.64 \%$ to $21.28 \%$ ). Among the persons with prediabetes ( $n=12)$, three had both IFG and IGT and nine had IGT (Table-7).

The tabulated sensitivity (true positive rate) of the IDRS cut-off score $\geq 60$ (high risk classification) was found to be $84.21 \%$ with a specificity (true negative rate) of $55.24 \%$. The false positive rate and false negative rate was $44.76 \%$ and $15.79 \%$ respectively. The positive predictive value was $20.0 \%$ and negative predictive value was $96.34 \%$ (Table-8).

On ROC curve analysis of IDRS cut-offs for the identification of persons with 'raised blood glucose', the 50 and above score had a $100 \%$ sensitivity with a low specificity of $27.27 \%$ and an accuracy of $35.80 \%$. Increasing the cut-off to 70 and above, raised the specificity to $86.01 \%$ with a decrease in the sensitivity to $31.58 \%$. The IDRS recommended cut-off of 60 and above was found a sensitivity and specificity of $84.21 \%$ and $55.24 \%$ with an accuracy of $58.64 \%$. The positive likelihood ratio was 1.88 and the negative likelihood ratio was 0.28 at this cut-off (Table-9).

The IDRS ROC AUC for a score of 60 and above was 0.69 (95\% CI 0.62 to 0.77 ) as compared to 0.87 for FPG and 0.98 for the gold standard $2 \mathrm{hr}$ PG for the identification of persons with raised blood glucose (Fig. 1).

\section{DISCUSSION}

In the present study, IDRS identified just under half of the screened population (46.05\%) as high risk for developing diabetes. In comparison, in a study carried out in a semi-urban area near Chennai, India ( $n=304)$ about $37 \%$ were found to be at high risk. The lower age ( 41.5 years vs 49.15 years) may have accounted for the lower proportion. ${ }^{14}$

The association between diabetes and hypertension has been well documented in literature. ${ }^{15,16}$ The positive association of IDRS value 60 and above with hypertensive status as seen in the present study may add to the utility of screening with IDRS.

The definitive testing yielded a prevalence of undiagnosed diabetes which ranged between 1.75\% to $8.70 \%$. The prevalence of prediabetes was higher at about $3.89 \%$ to $12.58 \%$. In comparison with the prevalence of known diabetes (50/306) during the screening period, about one in eight persons with diabetes was found to be undiagnosed. Prevalence of undiagnosed diabetes was lower than the global estimates of 1 in 2 persons. The higher yield of prediabetes as compared to diabetes also suggests an increased awareness about diabetes among the screened population. As the yield of raised blood glucose in the present study was small, further linear or logistic regression analysis was not performed.

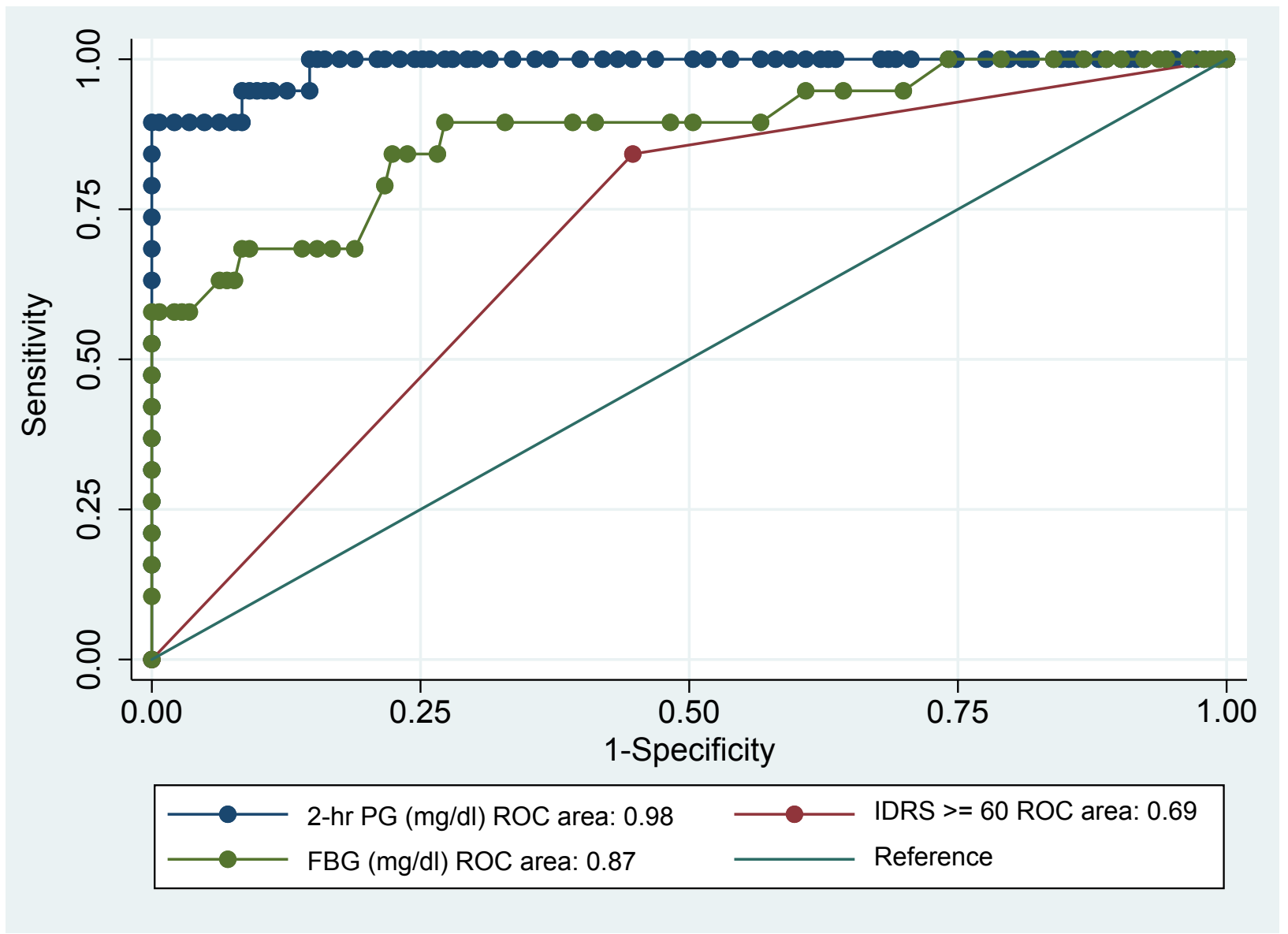

Fig 1: ROC area curve of IDRS against gold standard 2-hour plasma glucose and fasting plasma glucose for the identification of diabetes and prediabetes 
A similar study was carried out in Mangalore, India among 551 adults. The response rate for OGTT was higher than that of the present study $(68.9 \%$ vs $63.28 \%$ ). The study reported a sensitivity of $62.2 \%$ and a specificity of $73.7 \%$ for the IDRS cut-off $\geq 60$. In comparison, the sensitivity in our study was higher at $84.21 \%$ with a lower specificity of $55.24 \% .{ }^{17}$ It would be appropriate to argue here that a screening test would need to be more sensitive than specific as found in the present study. ${ }^{6}$

In another IDRS validation study among adults in an urban slum in India ( $\mathrm{n}=155)$, Dudeja et al reported an IDRS sensitivity and specificity of $95.12 \%$ and $28.95 \%$ respectively. This study however confirmed diabetes using only the fasting blood sugar testing. ${ }^{18}$

The World Health Organization (WHO) and the International Diabetes Federation (IDF) have recommended that OGTT be used for the diagnosis of diabetes. The American Diabetes Association (ADA) however differed and suggested the use of FPG in the diagnosis of diabetes. ADA has also lowered the cut-off of IFG to $100 \mathrm{mg} / \mathrm{dl}$ from $110 \mathrm{mg} / \mathrm{dl}^{19,20}$ In the present study, a majority of the 'raised blood glucose' yield was due to the post glucose 2-hr PG testing.

Another community based validation study carried out in an urban North Indian population $(n=615)$ reported that an IDRS score of 60 and above had a lower sensitivity of $45.5 \%$ and a higher specificity of $88.0 \% .^{21}$

Likelihood ratios were reported to assess the potential utility of IDRS. The positive likelihood ratio $(\mathrm{LR}+)$ was the ratio of true positives to false positives. $\mathrm{LR}+$ of about 2 was interpreted as an increase in the probability of diabetes or prediabetes by $15 \%$ for a person with an IDRS score of 60 or more. On the other hand, negative LR (LR-) was the ratio of false negatives to true negatives. LR- of 0.32 was interpreted as a $30 \%$ decrease in the probability of diabetes or prediabetes for an individual with an IDRS score less than $60 .{ }^{22}$

Non-invasive risk stratification tools have been recommended as a cost-effective method of screening for diabetes and prediabetes. The present study findings suggest that the IDRS may be an appropriate tool to screen for diabetes and prediabetes among the adult population in Kathmandu, Nepal. With a negative predictive value of about $97 \%$, the IDRS may also be useful in a clinical setting to decide which individual may or may not need definitive testing. This may help to reduce the out of pocket (OOP) expenditure for the individual in a low- and middle-income nation such as Nepal. ${ }^{23}$

However, IDRS may have overestimated the actual risk of diabetes as seen by the $44.76 \%$ false positive rate. Another concern raised was, 'how likely were the persons with prediabetes to have developed diabetes'? A meta-analysis of observational studies among persons with prediabetes reported that the progression to a state of diabetes was estimated to be 47 per 1000 person years for persons with IFG,
56 per 1000 person years for IGT and 76 per 1000 person years for both IFG and IGT. ${ }^{24,25}$ This suggested that persons with an impairment of both fasting as well as post-prandial glucose levels were more likely to develop diabetes in the near future. IDF guidelines suggest that persons who screen negative should be retested after 3-5 years. ${ }^{3}$ A follow up testing of the cohort of 'false positive' persons over the next 5-10 years would probably best answer this concern.

Strengths of the study: The community-based study design to screen for undiagnosed diabetes, the step wise approach including the non-invasive tool and estimation of RCBG and the use of both FPG and the $2 \mathrm{hr}$ PG following a 75g OGTT to identify diabetes and prediabetes are the main strengths of this study.

Limitations of the study: A community-based screening program can attract persons who have the health condition, those with a propensity to seek health care or who are more interested in their health which can introduce a selection bias. We tried to minimize this bias by screening about $43.33 \%$ of the households in the community to secure a representative sample $(n=304)$. Persons with diabetes were eager participants in the screening program $(n=50)$; while offering them testing facilities, they were excluded from the data analysis for the IDRS performance. Response rate for the definitive testing was $63.28 \%$ among those without diabetes $(n=256)$ and yielded a number which was well over the required sample size. However, a greater response may have improved the yield of undiagnosed diabetes in the screened population.

In conclusion, the IDRS value of 60 and above predicted the overall risk of diabetes and prediabetes with a sensitivity of $84.21 \%$ and specificity of $55.24 \%$ among the screened population. The persons with screen-detected diabetes were offered treatment and care at our tertiary health care facility. Screening with IDRS also provided an opportunity for primary prevention among persons with prediabetes or a high-risk classification focusing on diet, physical activity and weight management. This may help to prevent or delay the progression to diabetes among these persons. IDRS may be a suitable screening tool for diabetes and prediabetes in the adult Nepalese study population.

\section{REFERENCES}

1. World Health Organization. Global Report on Diabetes. 2016; 88.

2. Heuck C-C, Home PD, Reinauer H, et al. Laboratory Diagnosis and Monitoring of Diabetes Mellitus. World Health Organ 2002; 1-26.

3. International Diabetes Federation Guideline Development Group. Global guideline for type 2 diabetes. Diabetes Res Clin Pract 2014; 104: 1-52.

4. Flórez H. Steps toward the primary prevention of type II diabetes mellitus. Various epidemiological considerations. Investig clínica 1997; 38: 39-52. 
5. WHO. Definition and Diagnosis of Diabetes Mellitus and Intermediate Hyperglycemia. Who2 2006; 50.

6. Harris MI. Classification, Diagnostic Criteria, and Screening for Diabetes. Diabetes Am 2nd 1995; 18.

7. Abbasi A, Peelen LM, Corpeleijn E, et al. Prediction models for risk of developing type 2 diabetes: systematic literature search and independent external validation study. Br Med J [ Internet] 2012 [cited 2017 Aug 30]; 345 Available at: http://www. bmj.com/content/345/bmj.e5900.abstract.

8. Somannavar S, Ganesan A, Deepa M, et al. Random capillary blood glucose cut points for diabetes and pre-diabetes derived from community-based opportunistic screening in India. Diabetes Care 2009; 32: 641-3.

9. Buijsse B, Simmons RK, Griffin SJ, et al. Risk assessment tools for identifying individuals at risk of developing type 2 diabetes. Epidemiol Rev 2011; 33: 46-62.

10. Mohan V, Deepa R, Deepa M, et al. A simplified Indian Diabetes Risk Score for screening for undiagnosed diabetic subjects. J Assoc Physicians India 2005; 53: 759-63.

11. Gyawali B, Sharma R, Neupane D, et al. Prevalence of type 2 diabetes in Nepal: a systematic review and meta-analysis from 2000 to 2014. Glob Health Action 2015; 8: 10.3402/gha.v8.29088.

12. Khunti K, Mani H, Achana F, et al. Systematic Review and Meta-Analysis of Response Rates and Diagnostic Yield of Screening for Type 2 Diabetes and Those at High Risk of Diabetes. PLoS One 2015; 10: e0135702.

13. World Health Organization. The WHO STEPwise approach to noncommunicable disease risk factor surveillance. World Health Organ 2017; 1-474.

14. Nagalingam S, Sundaramoorthy K, Arumugam B. Screening for diabetes using Indian diabetes risk score. Int J Adv Med 2016; 415-418.

15. Epstein M, Sowers JR. Diabetes mellitus and hypertension. Hypertens (Dallas, Tex 1979) 1992; 19: 403-418.
16. Sampanis C, Zamboulis C. Arterial hypertension in diabetes mellitus: from theory to clinical practice. Hippokratia 2008; 12: 74-80.

17. Adhikari P, Pathak R, Kotian S. Validation of the MDRF-Indian Diabetes Risk Score (IDRS) in another south Indian population through the Boloor Diabetes Study (BDS). J Assoc Physicians India 2010; 58: 434-6.

18. Dudeja P, Singh G, Gadekar T et al. Performance of Indian diabetes risk score (IDRS) as screening tool for diabetes in an urban slum. Med J Armed Forces India 2017; 73: 123-128.

19. WHO. Definition,Diagnosis and Classification of Diabetes Mellitus. WHO/NCD/NCS/99.2 pdf 1999; 1-59.

20. American Diabetes Association. Standards of Medical care in diabetes - 2017. J Clin Appl Res Educ 2017; 40: 1-142.

21. Agarwal AK, Ahirwar G, Marskole $\mathrm{P}$, et al. A community based study to assess the validity of Indian diabetic risk score, among urban population of North Central India. Int J Community Med Public Heal Vol 4, No 6 June 2017DO - 1018203/2394-6040. ijcmph20172184 Available at: http://www.ijcmph. com/index.php/ijcmph/article/view/1331 (2017).

22. McGee S. Simplifying likelihood ratios. J Gen Intern Med 2002; 17: 647-650.

23. Khunti K, Gillies CL, Taub NA, et al. A comparison of cost per case detected of screening strategies for Type 2 diabetes and impaired glucose regulation: modelling study. Diabetes Res Clin Pract 2012; 97: 505-13.

24. Loveman E, Frampton G, Clegg A. The clinical effectiveness of diabetes education models for Type 2 diabetes: a systematic review. Health Technol Assess (Rockv); 12. Epub ahead of print April 2008. DOI: $10.3310 /$ hta12090.

25. Morris DH, Khunti K, Achana F, et al. Progression rates from HbA1c 6.0-6.4\% and other prediabetes definitions to type 2 diabetes: a meta-analysis. Diabetologia 2013; 56: 1489-93. 